\title{
Belphégor
}

\section{Charles Grivel ou le dispositif de la marge}

Petites réflexions sur les couvertures populaires illustrées

\section{Marie-Ève Thérenty}

\section{(2) OpenEdition \\ Journals}

Electronic version

URL: https://journals.openedition.org/belphegor/1248

DOI: $10.4000 /$ belphegor. 1248

ISSN: 1499-7185

Publisher

LPCM

Electronic reference

Marie-Ėve Thérenty, "Charles Grivel ou le dispositif de la marge ", Belphégor [Online], 16-1 | 2018, Online since 16 July 2018, connection on 19 October 2021. URL: http://journals.openedition.org/belphegor/ 1248 ; DOI: https://doi.org/10.4000/belphegor.1248

This text was automatically generated on 19 October 2021 .

\section{(c)}

Belphégor est mis à disposition selon les termes de la Licence Creative Commons Attribution - Pas d'Utilisation Commerciale - Pas de Modification 4.0 International. 


\section{Charles Grivel ou le dispositif de la}

\section{marge}

Petites réflexions sur les couvertures populaires illustrées

\section{Marie-Ève Thérenty}

1 Je ne faisais pas partie du premier cercle de Charles Grivel mais, lorsque je me retourne, je le découvre présent à chaque moment significatif de mon parcours académique, par exemple lors du colloque Presse et littérature au XIXe siècle à Montpellier en 2001 qui engageait tout le projet de La Civilisation du journal, de Medias 19 et de Numapresse ou lors de la préparation du numéro de Romantisme sur l'œuvre-monde, phénomène littéraire qui me tient à cœur. Mais je me souviens surtout pendant la préparation de ma thèse de ma longue quête de Production de lintérêt romanesque ${ }^{1}$, son ouvrage systématiquement cité dans les travaux les plus pertinents mais qui semblait avoir mystérieusement disparu de toutes les bibliothèques. Ce livre m'intéressait notamment parce qu'il repérait un corpus romanesque massif, annonçant les enquêtes systématiques d'un Marc Angenot ${ }^{2}$, les expérimentations d'un Franco Moretti ${ }^{3}$ et bien sûr les fouilles de données sur grands corpus numérisés ${ }^{4}$. Quand le livre apparaissait au catalogue d'une bibliothèque, et lorsque l'on en faisait la demande, immuablement on voyait arriver des magasins le volume complémentaire, celui des annexes. Le corps même de l'ouvrage s'évaporait, m'évoquant un livre fantôme comme ce traité romantique sur l'incommodité des commodes de Jules Vabre, parfois cité mais jamais écrit. Le nom même de l'éditeur, Mouton, et la double localisation Paris/La Haye ajoutaient encore à l'impression de mystification. Craignant l'accusation de lacune impardonnable et contribuant sans doute ainsi à l'égarement de futurs doctorants, j'avais quand même placé l'ouvrage inaccessible dans ma bibliographie de thèse. Lorsque j'ai rencontré pour la première fois Charles Grivel, après la soutenance, je lui avais détaillé mon insatiable quête de son livre et l'impression de canular que j'en avais tirée. Il avait ri largement, comme il savait le faire, m'avait raconté une histoire obscure de réédition et m'avait offert obligeamment de m'envoyer Production de l'intérêt romanesque en double exemplaire, l'un pour ma bibliothèque personnelle, l'autre pour celle de mon université. Par la poste était arrivé quelques semaines plus tard un paquet soigneusement enveloppé contenant deux exemplaires ... du volume complémentaire 
de Production de l'intérêt romanesque. Tout lecteur peut maintenant avoir une idée du volume complémentaire puisque Belphégor l'a récemment réédité, contribuant à la légende d'un ouvrage académique essentiel mais dont on ne peut se procurer facilement que les annexes, les marges. Nul hasard cependant, nous le verrons, dans ce geste finalement très grivellien.

2 Les nombreux et éclectiques travaux de Charles Grivel manifestent un certain nombre de principes à mes yeux salutaires : refus d'isoler le corpus canonique de la littérature du massif des œuvres populaires, médiatiques et même de la production culturelle de masse ; nécessité de repenser la littérature dans son contexte de production et donc de désolidariser son étude de celle du livre qui n'en constitue qu'une incarnation historique ; intérêt pour les dispositifs éditoriaux et médiatiques et notamment pour le livre illustré. Il est évidemment significatif que beaucoup d'articles de Charles Grivel portent sur la photographie tant ce paradigme a modifié durablement les régimes de représentation sur lesquels a vécu la littérature et le rapport à la fois au réel et au régime sériel. La fascination de Charles Grivel pour l'hybride sensible à travers la question du livre illustré était une prescience de l'évolution de la littérature entraînée aujourd'hui inéluctablement vers la prise en compte de la transmédialité et l'intermédialité.

3 Relisant son article sur les couvertures illustrées du roman populaire ${ }^{5}$, article qui correspond à une communication effectuée en 2002 dans le cadre des travaux organisés sur les littératures populaires par l'université de Limoges, sous la houlette de Jacques Migozzi, je suis frappée par la manière dont Charles Grivel définit en creux un nouveau paradigme pour les études littéraires qui va faire florès dans les années qui vont immédiatement suivre. Le geste était encore transgressif au début du millénaire d'abord parce que le roman policier ne faisait généralement pas partie du périmètre d'un chercheur académique en littérature ${ }^{6}$ mais surtout parce que la couverture, élément du paratexte par excellence, échappe à l'auteur et relève des champs de compétence conjoints de l'éditeur et de l'illustrateur. Comme souvent, Charles Grivel exerçait son talent sur des sujets qui semblaient en marge de la marge. Se plaçant du côté de la réception, Grivel rappelait pourtant que la couverture constitue la première interface entre le lecteur et le livre et que la littérature appartient à la sphère des productions culturelles inscrites dans l'ère de la consommation de masse développée depuis le milieu du XIX ${ }^{e}$ siècle. Remettre la marge au centre lui semblait donc un geste heuristique et salutaire.

4 Dès le début de l'article, Charles Grivel faisant l'état de l'art de son objet égrène une série de noms, tous chercheurs curieux et confirmés, venant d'horizons variés mais qui ont contribué à introduire de l'hybridité et de la pluridisciplinarité dans les études littéraires. Il se dégage ainsi une parentèle académique qui permet de le situer, dessine un compagnonnage particulièrement éclairant d'universitaires qui, comme lui, sont des transfuges et des innovants. De la poétique (Gérard Genette et Philippe Hamon) aux sciences de l'information et de la communication (Marc Lits) via les sciences sociales et l'histoire (Anne-Marie Thiesse), de Limoges à Louvain en passant par Paris, telle était la géographie universitaire de Grivel. Partout où cela bougeait du côté des supports, des visual studies, de la littérature médiatique et populaire, Grivel répondait présent.

5 L'article est organisé en microséquences numérotées. La séquence permet d'avancer pas à pas, en se répétant un peu à chaque fois - «je reprends »-, de tester plusieurs versions de la même idée. L'article fournit ainsi comme une propédeutique de sa 
discipline. Charles ne répugne ni à la première personne, ni à la convocation de l'antonomase audacieuse: "Je vois ce qui se passe au fond du corridor des lettres. Descendu là par une bouche propice, je me déplace, tel Marius dans les égouts de Paris, je me désole comme le pantin devant sa grille à considérer là hors de portée les Paquita de tous les romans ${ }^{7}$ ». Car Charles Grivel tenait de la tradition des universitaires qui écrivent leurs articles. Il aimait le jeu des mots. Lorsqu'il cite Gérard Genette, ce sont ses formules humoristiques qu'il traque, ayant reconnu, avant même Bardadrac ${ }^{8}$ derrière le poéticien sérieux l'humoriste impénitent. Grivel, aussi, cherchait la formule qui fait mouche, définissant par exemple avec bonheur la couverture du roman policier comme un "bâillon typographique illustré 9 " imposé au livre. Plus loin, la couverture montre «toujours un peu trop et toujours pas assez. Le rapport est celui d'une robe à un corps pour l'amant». Cette nouvelle image est révélatrice de la perversité de la couverture à la fois dévoilement et dissimulation, voire tromperie. La métaphore se précise, de plus en plus grivellienne, c'est-à-dire grivoise, grisée: l'image de la couverture est comme "cette barrière de chair blanche qu'une jarretière opportunément découverte découpe au bon moment sous cela qu'on ne verra pas ${ }^{10}$ ». Rien de gratuit dans ces images sexuelles. Charles Grivel cherche à nous faire comprendre que la lecture est provoquée par un désir motivé par la «scène primitive $^{11}$ » que constitue la couverture. Ces images permettent aussi de préciser que la couverture est un faux, elle ment comme le coupable dans le roman policier qu'elle recouvre. Car cette scène primitive ne figure pas dans le livre. Grivel développe, pour notre plus grand plaisir, une forme de mimétisme entre l'objet qu'il étudie, le roman policier et son propre discours. On est prêt à convoquer le détecteur de mensonges pour faire avouer la couverture...

6 S'inscrivant ainsi dans le sillage de Gérard Genette et de son paradigmatique Seuils, sur un mode prétéritif et léger - «je ne fais que débroussailler »-, Charles Grivel énumère les fonctions possibles de la couverture: la reliure, la protection, l'information, l'intégration dans une série ou dans une collection, la dimension projective et rétensive grâce à l'illustration, et il fait aussi, de manière toujours apparemment désinvolte, un survol finalement précis de l'histoire de la couverture, montrant ainsi sa sensibilité à la poétique historique des supports. Mais il voit surtout la couverture, « mécanisme visuel de visionnement" comme un dispositif: "la couverture illustrée, battement d'incertitude imposée dès l'abord à la matière écrite, constitue ainsi le livre en un mécanisme pervers ${ }^{12} \%$. La couverture montrerait une scène cachée, une scène impossible, une « scène primitive ", qui n'apparaît jamais, et pour cause, dans le roman. Le lecteur court donc après cette image, série après série, et l'intérêt romanesque se fonderait en grande partie sur ce suspense narratif. Comme Grivel sait que les paradoxes sont au cœur de la mécanique de l'article, il n'hésite pas à proclamer que la lecture se fait rétroactivement et pour confirmation de l'image.

7 Cette éblouissante mise au point se termine par l'examen d'un cas : l'ensemble de la production romanesque des éditions Fleuve noir. C'est l'occasion de feuilleter un gigantesque livre d'images, celles produites par Michel Gourdon, le Starace du fleuve noir.

8 La critique littéraire, comme la littérature, ne progresse pas mais revendique des changements de paradigme que l'on estime fondamentaux. Sans doute ce lecteur fantasmé par Grivel qui lit le livre à la recherche de son illustration capitale manque-til aux yeux de la sociologie de la réception de 2018, d'un peu d'incarnation. Aujourd'hui 
lorsqu'il s'agit d'usages, l'enquête est préconisée, même en littérature, et les sociologues ${ }^{13}$ nous enjoignent à un peu de prudence dans la projection d'un lecteur. Les nombreux témoignages de lecteurs du Fleuve noir et d'admirateurs de Gourdon qui figurent sur la toile aujourd'hui montrent la force d'un imaginaire qui s'est aussi construit sur un réseau de distribution particulier : un témoin raconte ainsi que dans les années 70 dans la boulangerie de son petit village, on trouvait outre le pain un tourniquet du Fleuve noir ${ }^{14}$. Les lecteurs corroborent l'attraction des couvertures - « Il m'arrive maintenant d'acheter un livre Fleuve noir d'abord pour l'illustration de Gourdon ${ }^{15} »-$ mais insistent plutôt dans leurs déclarations sur l'idée de série, selon le principe même d'ailleurs qui pousse Grivel à juxtaposer les illustrations. Les illustrations de Gourdon se goûtaient en fait quasiment indépendamment des livres par l'effet de sérialité qu'elles construisaient. Elles permettaient d'élaborer une forme de " signalétique ${ }^{16}$ ". La recherche de cet effet justifie sans doute en partie les lacunes de la relation entre le texte et l'image - même si Gourdon se faisait bien raconter l'histoire par l'éditeur et souvent obtenait les coordonnées de l'auteur pour s'entendre avec lui ${ }^{17}$ - et explique la scène absentée de la couverture. La scène absentée n'a pas besoin de se retrouver précisément dans le livre puisqu'elle est archétypale, architextuelle, dirait Matthieu Letourneux ${ }^{18}$. Les lecteurs, ou au moins ces amateurs éclairés que sont les collectionneurs, loin d'être systématiquement trompés par leurs fantasmes, paraissent avoir domestiqué le dispositif et l'avoir identifié comme architextuel et donc détaché de la lettre des histoires racontées. Il est en fait peu probable que le lecteur ordinaire soit leurré longtemps par la couverture. Les amateurs des images de Gourdon invoquent d'ailleurs d'autres séries et d'autres médias que le livre pour expliquer leur attachement aux illustrations : friands des ressemblances que Gourdon soignait entre les personnages des couvertures et les acteurs, ils collectionnent ces couvertures aussi pour leur sérialité avec le cinéma. La couverture s'avère donc exemplaire de ces échanges transmédiatiques caractéristiques des pratiques culturelles contemporaines.

9 Cette remarque n'enlève rien à la pertinence de l'article de Grivel qui souligne effectivement un point essentiel : l'illustration de roman populaire fonctionne comme œil certes mais surtout comme voile ou comme obturateur. Cette idée d'une illustration tout sauf illustrative constitue une intuition forte, que l'on retrouve dans d'autres articles par la suite qui s'inscrivent, explicitement ou non, dans la lignée grivellienne. Amy Wigelsworth dans un article sur l'édition Rouff des Mystères de Paris montre que "l'illustration se révèle tout sauf illustrative ${ }^{19}$ ", ce qui est une reprise d'une antienne de Grivel $^{20}$. L'article de Matthieu Letourneux récemment paru sur les livraisons romanesques illustrées à la fin du $\mathrm{XIX}^{\mathrm{e}}$ siècle $^{21}$, insiste aussi sur le déport dès le XIX ${ }^{\mathrm{e}}$ siècle de la signification du roman vers l'éditeur et l'illustrateur: " chaque livraison apparaît comme un ensemble circonscrit défini par l'image liminaire, imposant sa propre signification, d'une façon qui n'a été voulue ni par l'écrivain, ni par l'éditeur, ni par le dessinateur». Il existerait donc une "énonciation éditoriale ${ }^{22}$ " collective, proposition qui se trouve aussi en creux dans l'article de Charles Grivel. Surtout Matthieu Letourneux montre bien que l'illusion référentielle est court-circuitée au profit de la série culturelle appuyée au XIX ${ }^{\mathrm{e}}$ siècle sur des stéréotypes dramatiques ou picturaux, au $\mathrm{XX}^{\mathrm{e}}$ sur des stéréotypes cinématographiques. J'ai tenté de montrer moimême ${ }^{23}$ que la grammaire illustrative des romans, fondée sur la répétition de trois genres d'images : la vue, la scène et le type, finissait par avoir une incidence à rebours sur la poétique des œuvres, qu'elles soient produites en série ou non d'ailleurs. Cette grammaire constitue une image du support avec laquelle les écrivains ont pu jouer 
parfois selon des effets-supports littérarisés dont il reste à faire l'histoire et la taxinomie. La remarque suivante de l'écrivain Serge Brussolo concernant la présentation matérielle des couvertures de Fleuve Noir - « ... et soudain, ce petit roman imprimé sur papier-cul, enveloppé dans une couverture d'une laideur totale m'électrocuta. Je ne m'en suis jamais guéri ${ }^{24}{ }^{4}$ - renvoie aussi à une poétique et à un imaginaire du support et prouve qu'il existe un effet de la matérialité des supports sur la création: les illustrations de Michel Gourdon ne provenaient pas peut-être de la diégèse des œuvres mais elles n'ont sans doute pas été sans incidence sur l'écriture du roman policier. Cela aussi d'ailleurs, d'une certaine manière, Grivel l'avait déjà dit et comme toujours latéralement dans un article périphérique et complémentaire au premier : « Notre thèse, de façon succincte, peut être présentée de la manière suivante : la popularisation du circuit littéraire entraine une illustration du texte (narratif surtout, encyclopédique aussi bien que romanesque) et celle-ci entraine à son tour un remodelage textuel caractérisé par le morcellement, l'accélération et l'allégorisation ${ }^{25}$ ».

En fait l'article sur les couvertures illustrées et la nébuleuse d'autres articles de Charles Grivel cités à la marge montrent combien Charles Grivel a anticipé puis accompagné un des paradigmes, à notre avis, les plus féconds de renouvellement des études littéraires au tournant des années 2000 autour du support. Ce paradigme s'est développé simultanément dans plusieurs espaces scientifiques de province ou de périphérie, à Limoges, à Nanterre, à Montréal, à Toulouse, à Liège, à Montpellier. Ces différents pôles lui ont donné des noms variés selon qu'ils faisaient porter l'accent sur le pôle de la réception, de la production ou de la consommation: culture matérielle, école de Toulouse ou école du dispositif, culture médiatique, poétique du support. Charles Grivel allant au bout de son geste théorique et manifestaire aurait peut-être préféré le terme de culture visuelle comme le montre l'article «Le Terrain des images » qui rappelait quelques-uns de ses objets hybrides de prédilection: le livre à vignettes, le roman populaire illustré, le journal illustré, le ciné-roman, le roman photo et qui tenait un discours de la méthode encore tout à fait d'actualité. « Nous ne sélectionnons pas (pas au premier chef) des " contenus ", des «thèmes ", des "motifs", du «sens ", mais des matérialisations, des effets de mise en scène, des techniques. Nous envisageons la force de l'instrument - car elle prime sur tout le répertoire. Cela implique que le champ de recherche inclut l'histoire des moyens de reproduction; elle se fera histoire des techniques et des croyances - outre ce que nous venons de dire -, plus qu'histoire des textes (au sens restrictif). Mais il est juste de dire aussi que ceux-ci contiennent le protocole des savoirs et qu'ils en sont la représentation et diffuse et controversée ». Ce programme recoupe en partie celui qu'avait mis en place l'école du dispositif de Toulouse - ce n'est pas un hasard si dans l'article sur les couvertures populaires, Grivel employait le mot. Il n'aurait sans doute pas reculé devant ces formules proposées par Philippe Ortel qui s'appliquent extraordinairement bien aux objets qui le fascinent : « le dispositif apparaît chaque fois qu'une structure ouvre sur de la conjoncture ${ }^{26} »$. Le dispositif c'est à la fois une couverture qui fait lire un livre à la recherche de la scène liminaire ; ce pourrait être aussi un livre clé qui renvoie inlassablement à son volume d'annexes. Comme on collectionne les illustrations de Gourdon sans lire les romans policiers, on finit par ne lire que le supplément, voire à le rééditer détaché du volume principal comme l'a fait Belphégor, qui a compris que dans Production de l'intérêt romanesque, comme souvent chez Grivel, l'essentiel vient de la marge ${ }^{27}$. 


\section{NOTES}

1. Charles Grivel, Production de l'intérêt romanesque, La Haye-Paris, Mouton, 1973.

2. Voir Marc Angenot, 1889, un état du discours social, réédité sur le site medias19, http:// www.medias19.org/index.php?id=11003

3. Franco Moretti, Atlas du roman européen (1800-1900), Seuil, 2000.

4. Franco Moretti (dir.), La Littérature au laboratoire, Ithaque, 2016. Voir aussi pour la presse française, www.numapresse.org

5. Charles Grivel, «De la couverture illustrée du roman populaire », Production(s) du populaire, Limoges, Presses universitaires de Limoges, Médiatextes, 2005, p. 281-305.

6. Bien sûr le lecteur pense alors à Jacques Dubois et son Roman policier et la modernité, paru chez Nathan en 1992 mais Jacques Dubois, comme Charles Grivel d'ailleurs et comme beaucoup d'autres en Belgique, en Suisse et au Québec par exemple, a la liberté de celui qui n'écrit pas depuis l'hexagone.

7. Op. cit., p. 282.

8. Gérard Genette, Bardadrac, Seuil, 2011.

9. Op. cit., p. 283.

10. Op. cit., p. 291.

11. Car même si Grivel s'en revendique rarement explicitement, il emprunte aussi à la psychanalyse, dans un éclectisme productif et jamais exclusif.

12. Ibid.

13. Je pense aux travaux récents de Cécile Rabot et de Gisèle Sapiro mais aussi à un essai d'Annie Collovald et d'Erik Neveu, Lire le noir. Enquête sur les lecteurs de récits policiers, Presses universitaires de Rennes, 2016.

14. Voir le blog de Claude Le Nocher, Action-Suspense, billet du 7 juin 2011, http://www.actionsuspense.com/article-le-polar-de-1950-a-1980-des-romans-populaires-75937861.html

15. Voir le blog « Le boudoir de Zigomar » et le billet du 26 janvier 2012 « Michel Gourdon, 30 ans sur le fleuve noir", https://leboudoirdezigomar.wordpress.com/2012/01/26/michelgourdon-30-ans-sur-le-fleuve-noir/

16. Annie Collovald et Erik Neveu, op. cit., p. 133.

17. Voir « les tronches de Michel Gourdon », http://www.inspecteurmatis.com/sp/tronches.htm

18. Je renvoie au livre de Matthieu Letourneux, Fictions à la chaîne, Seuil, 2017.

19. Amy Wigelsworth, «Illustration et dissimulation dans Les Mystères de Paris, enjeux de l'édition illustrée », Romantisme, 2017/1, n 175, p. 97-112.

20. Voir par exemple « l'illustration n'est jamais illustrative dans Charles Grivel, « Comment voiton ? Illustration, non-illustration », Les Cahiers naturalistes, 1992, n 66, p. 137.

21. «Illustration et sérialité dans les livraisons romanesques » (1870-1910), Vincent Chambarlhac et Bertrand Tillier, Coups de crayons sous la Troisième République, Le Murmure, 2017.

22. Voir par exemple Emmanuël Souchier, "Formes et pouvoirs de l'énonciation éditoriale", Communications et langages, $\mathrm{n}^{\circ}$ 154, décembre 2007, p. 23-38.

23. Marie-Ève Thérenty, "Scènes et types. Poétique du roman de mœurs et grammaire de l'illustration" dans Philippe Dufour, Bernard Gendrel, Guy Larroux, Le Roman de mours, Classiques Garnier, à paraître en 2018.

24. Déclaration de Serge Brussolo qui montre d'ailleurs que cet imaginaire n'a peut-être pas été sans influence sur la pratique des auteurs.

25. Voir Charles Grivel, "Le terrain des images ", In : Bulletin Zéro. Coordination des Chercheurs en Littératures populaires et Culture médiatique, Publication de l'Université de Mannheim, 
Romanistik I, septembre 2000, p. 39-49 repris à l'adresse suivante: https:// dalspace.library.dal.ca/bitstream/handle/10222/47640/01_01_Grivel_Images_cont.pdf

26. Philippe Ortel, La Littérature à l'ère de la photographie, Enquête sur une révolution invisible, Chambon, 2002, p. 346 cité par Bernard Vouilloux, «Du dispositif», dans Philippe Ortel (dir.), Discours, image, dispositif. Penser la représentation II, L'Harmattan, 2008, p. 19.

27. Charles Grivel, Production de l'intérêt romanesque, éditions en ligne, Belphégor, inédit, https:// dalspace.library.dal.ca/bitstream/handle/10222/47786/09_02_Grivel_prod_cont.pdf?

sequence $=1$

INDEX

Mots-clés: Grivel, illustrations, sérialité, littérature populaire, culture médiatique

\section{AUTHOR}

\section{MARIE-ÈVE THÉRENTY}

RIRRA21, Université Paul Valéry- Montpellier 3 